\title{
Validação do Inventário de Atitudes frente à Dor Crônica - Profissionais
}

\author{
VALIDATION OF THE SURVEY OF PAIN ATTITUDES - PROFESSIONALS
}

VALIDACIÓN DEL INVENTARIO DEACTITUDES FRENTE AL DOLOR CRÓNICO-PROFESIONALES

\section{Dayse Maioli Garcia', Cibele Andrucioli de Mattos Pimenta", Diná de Almeida Lopes Monteiro da Cruz"I}

\section{RESUMO}

Crenças influem no modo de as pessoas interpretarem os eventos e se conduzirem frente a eles. $\mathrm{O}$ objetivo deste estudo foi validar instrumento para identificar as crenças/atitudes de profissionais de saúde frente à dor crônica. $\mathrm{O}$ Inventário de Atitudes frente à Dor-Profissionais, adaptado do Inventário de Atitudes frente à Dor-Breve (IAD-Breve) utilizado para doentes , foi respondido por 75 profissionais de Centros de Dor (idade média = 42,8 anos; tempo médio após graduação = 16 anos; médicos = 58,7\% e fisioterapeutas $=42,7 \%$; com especialização com maior grau de formação = 42,7\% e mestrado/ doutorado $=26,7 \%)$. O IADProfissionais foi validado com 20 itens e a análise fatorial confirmou 6 domínios (emoção, controle, incapacidade, solicitude, cura médica e dano físico). A confiabilidade dos domínios variou entre 0,567 a 0,807 (alfa de Cronbach). Disponibiliza-se instrumento que auxiliará na organização de ações educativas.

\section{DESCRITORES}

Dor.

Atitude.

Psicometria.

\section{ABSTRACT}

Beliefs can influence the way people interpret events and behavior towards them. The aim of this study was validating an instrument to identify professionals' beliefs/attitudes towards chronic pain. The Survey of Pain Attitudes-Professionals (IAD-Profissionais), adapted from the Survey of Pain Attitude-Brief used for patients (SOPA-B), was answered by 75 professionals from Pain Clinics (mean age $=42.8$ years; mean years since graduation = 16 years; physicians = 58.7\%; physical therapists $=14.7 \%$; with specialization courses $=42.7 \%$; with Master's Degree/PhD = 26.7\%. The Survey of Pain Attitude-Professionals was validated with 20 items; the factorial analysis confirmed 6 domains (emotion, control, disability, solicitude, medical cure and harm). The reliability of the domains ranged from 0.567 to 0.807 (Cronbach's alpha). An instrument is available to help organizing educative actions.

\section{KEY WORDS}

Pain.

Attitudes.

Psychometrics.

\section{RESUMEN}

Las creencias influyen en el modo en que las personas interpretan los eventos y se conducen frente a ellos. El objetivo de este estudio fue validar un instrumento para identificar las creencias/actitudes de profesionales de salud frente al dolor crónico. El Inventario de Actitudes frente al Dolor - Profesionales, adaptado del Inventario de Actitudes frente al DolorBreve (IAD-Breve) utilizado para enfermos, fue respondido por 75 profesionales de Centros de Dolor (edad media = 42,8 años; tiempo medio después del pregrado $=16$ años; médicos $=58,7 \%$ $\mathrm{y}$ fisioterapeutas $=42,7 \%$; con especialización com mayor grado de formación = 42,7\% y maestría/ doctorado=26,7\%). El IADProfesionales fue validado con 20 ítems y el análisis factorial confirmó 6 dominios (emoción, control, incapacidad, solicitud, cura médica y daño físico). La confiabilidad de los dominios varió entre 0,567 a 0,807 (alfa de Cronbach). Está a disposición un instrumento que auxiliará en la organización de acciones educativas.

\section{DESCRIPTORES}

Dolor.

Actitud.

Psicometría.
I Mestre em Enfermagem pela Escola de Enfermagem, Universidade de São Paulo (EEUSP). Enfermeira do Grupo de Dor do Hospital SírioLibanês, São Paulo, SP, Brasil. enf_dayser@ yahoo.com.br

II Enfermeira. Professora Titular do Departamento de Enfermagem MédicoCirúrgica da Escola de Enfermagem, Universidade de São Paulo (EEUSP), São Paulo, SP, Brasil. parpca@usp.br

III Enfermeira. Professora Titular do Departamento de Enfermagem MédicoCirúrgica da Escola de Enfermagem, Universidade de São Paulo. Vice-Diretora da Escola de Enfermagem, Universidade de São Paulo (EEUSP), São Paulo, SP, Brasil. 


\section{INTRODUÇÃO}

O conhecimento e as crenças interferem na avaliação e nos tratamentos que os profissionais propõem. Cuida-se do que se conhece e se valoriza, do modo que se acredita ${ }^{(1-11)}$.

As crenças relacionadas à dor oncológica e dor aguda estão bem descritas na literatura, sob o ponto de vista do doente e dos profissionais. Os profissionais temem o uso dos opióides pelo medo do vício e depressão respiratória, julgam que a dor é acompanhante natural do processo e que seu controle é difícil (câncer) ou não é prioritário (dor aguda), entre outras. Essas concepções inadequadas são apontadas como razões para o inadequado controle da dor oncológica e da dor aguda que ainda se observa $a^{(1,3-4,6-10)}$. No entanto, as crenças dos profissionais de saúde sobre dor crônica não oncológica são ainda pouco conhecidas. Não há, em nosso meio, instrumento para a avaliação das crenças/atitudes dos profissionais de saúde sobre dor crônica não oncológica.

Considerando-se que dor crônica não oncológica apresenta etiopatogenia e proposta terapêutica muito diferente da dor aguda e da dor oncológica, que dor crônica é muito freqüente na população geral, é causa de sofrimento, de perdas financeiras e sociais, que a resposta aos tratamentos tradicionalmente propostos é modesta e que há estudos na literatura internacional que descrevem conceitos e crenças inadequadas dos profissionais, julgou-se desejável estudo que disponibilizasse instrumento em português para avaliar as crenças/atitudes dos profissionais sobre dor crônica.

O modelo de dor crônica não oncológica prevê que elementos físicos, cognitivos, emocionais e comportamentais determinam a vivência de dor ${ }^{(12-13)}$. Dor crônica persiste, de modo intermitente ou contínuo, por um tempo além do razoável para a cura de uma lesão, mas pode ou não estar relacionado a uma lesão tecidual e freqüentemente o comportamento de queixa de dor é desproporcional à lesão(2,12-13). A persistência da dor leva a modificações do aparelho neurológico e psíquico que se manifestam por meio de depressão, ansiedade, pensamentos catastróficos e comportamentos de incapacidade e dependência ${ }^{(13)}$. A terapia para dor crônica envolve o uso de medicamentos, meios físicos, ajuste de emoções e crenças ${ }^{(14)}$ e especial ênfase se dá para a identificação e ajuste das crenças.

Crenças são concepções muito antigas, culturalmente compartilhadas; são noções pré-existentes sobre a realidade que molda nossa percepção de nós mesmos, dos outros e de nosso ambiente; crenças são consideradas pelas pessoas como verdades absolutas ${ }^{(15)}$.
As crenças residem na cognição e predispõem a comportamentos ${ }^{(16)}$. Atitudes são disposições afetivas culturalmente aprendidas para reagir a algo (crenças, pessoas, instituições ou eventos), aproximando-se ou se afastando, aceitando ou negando, concordando ou discordando. É a preparação ou prontidão para a ação ${ }^{(15,17)}$.

O presente estudo teve por objetivo propor o Inventário de Atitudes frente à Dor-Profissionais, a partir de modificações do Inventário de Atitudes frente à Dor Versão Breve (IAD-Breve), utilizado para a avaliação de crenças/atitudes sobre dor crônica em doentes ${ }^{(18-19)}$. É a versão brasileira do Survey of Pain Attitudes-Brief (SOPA-B) ${ }^{(20)}$, um instrumento que avalia crenças/atitudes sobre dor crônica em doentes. O SOPA-B é a versão reduzida do instrumento Survey of Pain Attitudes (SOPA) ${ }^{(21)}$. O SOPA-B ${ }^{(20)}$ e o IAD-Breve ${ }^{(18-19)}$ são instrumentos com utilidade clínica e propriedades psicométricas adequadas.

O IAD-Breve (doentes) ${ }^{(18-19)}$ é um inventário auto-aplicado, com 30 itens, que avalia sete domínios de crenças/ atitudes de pacientes com dor crônica frente à dor: controle, emoção, incapacidade, dano físico, medicação, solicitude e cura médica.

O domínio controle refere-se a quanto o doente acredita que pode controlar sua dor; emoção refere-se a quanto o doente acredita que suas emoções influem na sua experiência dolorosa; incapacidade refere-se a quanto o doente acredita que está incapacitado pela dor; dano físico refere-se a quanto o doente acredita que a dor significa que ele está machucando a si mesmo e que deveria evitar exercícios físicos; medicação refere-se a quanto o doente acredita que medicamento é o melhor tratamento para dor crônica; solicitude refere-se a quanto o doente acredita que outros, especialmente os familiares, devem ser mais solícitos quando ele sente dor; cura médica refere-se a quanto o doente acredita na cura pela medicina para a sua dor. O instrumento é auto-aplicado, e os respondentes indicam a concordância com cada uma das assertivas, em escala tipo Likert de cinco pontos, que varia de 0 a $4(0=$ totalmente falso, $1=$ quase falso, $2=$ nem verdadeiro, nem falso, 3 = quase verdadeiro, $4=$ totalmente verdadeiro) $)^{(18-19)}$.

A análise do inventário é feita por domínio. O escore de cada domínio é calculado pela soma dos pontos das respostas de cada item, dividido pelo número de itens respondidos. O escore final médio de cada escala poderá variar entre 0 e 4 . Há itens invertidos $(4,8,11,23,24,26,27,28,29)$ que devem ter os seus escores revertidos antes da soma. A reversão do escore é feita subtraindo-se de 4 o escore escolhido pelo respondente (4 menos o escore escolhido). Não há pontos de corte, resposta certa ou errada, e os escores dos sete domínios ou escalas não são somados. Há orientações 
de respostas mais desejáveis por serem consideradas pelo autor do inventário como mais adaptativas. A orientação desejável dos escores para cada escala ${ }^{(18-21)}$ é: controle $=4$, emoção $=4$, incapacidade $=0$, dano físico $=0$, medicação $=0$, solicitude $=0$ e cura médica $=0$.

\section{MÉTODO}

\section{Participantes, período e local}

Entre agosto de 2005 e março de 2006 foram entrevistados os profissionais de Centros de Dor da cidade de São Paulo. Centro de Dor é um serviço que visa a tratar doentes com dor, constituído por médicos de várias especialidades, enfermeiros, fisioterapeutas, dentistas, psicólogos e assistentes sociais. No presente estudo foram incluídos todos Centros de Dor da cidade de São Paulo com pelo menos duas especialidades médicas e pelo menos dois tipos de profissionais (médicos e fisioterapeuta, médicos e enfermeiro ou médicos e psicólogo), por ser essa a configuração mínima de uma Clínica Multidisciplinar de Dor ${ }^{(22)}$.

A partir do site da Sociedade Brasileira para Estudo da Dor (www.dor.org.br), do livro História da Dor no Brasil ${ }^{(22)}$ e de informações de profissionais da área foram localizados onze Centros de Dor e nove aceitaram participar do estudo. Os Centros que aceitaram participar foram: Clinica da Dor do Hospital Nove de Julho, Centro de Tratamento da Dor do Hospital Sírio Libanês, Central da Dor do Hospital A.C. Camargo, Ambulatório de Dor do Hospital do Servidor Público Estadual, Clínica de Dor da Irmandade Santa Casa de Misericórdia de São Paulo, Serviço de Dor do Hospital Alemão Oswaldo Cruz, Ambulatório de Dor e Neurologia do Hospital das Clínicas da Faculdade de Medicina da Universidade de São Paulo (HC - FMUSP), Equipe de Controle de Dor Disciplina de Anestesiologia do HC - FMUSP e Unidade de Dor e Cuidados Paliativos do Instituto da Criança do HC-FMUSP (Processo CEP n. 462/2005).

\section{Instrumentos e procedimentos}

Os dados foram coletados por meio de dois instrumentos. O primeiro, denominado Identificação Pessoal, visou a caracterizar o profissional quanto aos dados sóciodemográficos e a experiência profissional no cuidado (avaliação e tratamento) a doentes com dor crônica não oncológica. O segundo instrumento foi o Inventário de Atitudes frente à Dor-Profissionais (Pré-teste), utilizado para identificar crenças/atitudes dos profissionais frente à dor crônica não oncológica.
Adaptação da versão Inventário de Atitudes frente à Dor profissionais

A adaptação inicial do IAD-Breve (doentes) para IADProfissionais (Pré-teste) foi realizada pelas autoras através da mudança das frases na primeira pessoa do singular (eu/meu) para a expressão no doente em vinte e nove assertivas, com exceção da assertiva 13 , que não faz referência à primeira pessoa do singular. Por exemplo: muitas vezes eu consigo influenciar a intensidade da dor que sinto para muitas vezes o doente consegue influenciar a intensidade da dor que sente.

Com essa modificação as autoras assumiram que as respostas para cada assertiva refletiriam as atitudes/crenças dos profissionais de saúde frente à dor crônica não-oncológica.

O instrumento foi submetido aos membros do Grupo de Pesquisa Dor, Controle de Sintomas e Cuidados Paliativos do Conselho Nacional para o Desenvolvimento Tecnológico e Científico (CNPq). Inicialmente, cinco juízas, enfermeiras participantes do grupo de pesquisa, responderiam ao questionário IAD-Profissionais (Pré-teste) e anotariam as dificuldades percebidas. A seguir, julgariam se cada uma das assertivas mantinha paralelo com a original e se permitiria ao profissional expressar sua crença. Porém, ao responderem o IAD-Profissionais (Pré-teste), as juízas perceberam que estavam expressando uma crença em relação ao doente e não sua própria crença em relação à dor crônica não-oncológica. Sugeriram, então, que a referência ao doente fosse retirada das assertivas. As decisões foram tomadas por consenso pelas pesquisadoras e juízas e os ajustes no instrumento foram feitos.

A proposição inicial foi de que o IAD-Profissionais (Préteste) manteria os mesmos domínios (7), número de assertivas (30), de categorias (escala tipo Likert com 5 categorias, de 0 a 4), os mesmos itens invertidos e mesmo método de obtenção do escore do IAD-Breve (doentes).

Assim, o domínio controle (a influência pessoal no controle da dor) está representado pelas questões 1, 12, 17, 20, e 22. O domínio emoção (relação entre emoção e intensidade da dor) está representado pelos itens 6, 10, 15, e 25. O domínio incapacidade (dor como incapacitante) está representado pelas questões 23, 26 e 30 do inventário. O domínio dano físico (dor como indicativo de dano tecidual) está representado pelos itens 11, 16, 19, 27 e 28. O domínio medicação (medicamentos são o melhor tratamento para dor crônica) está representado pelas questões 2, 5 e 13 do instrumento. $\mathrm{O}$ domínio solicitude (solicitude de outros frente à pessoa com dor) está representado pelos itens 3, 7, 9, 14 e 18. O domínio cura médica (cura por meios médicos) está representada pelas questões 4, 8, 21, 24, e 29.

Após essa fase, o inventário foi aplicado aos profissionais de saúde com vistas a se obter dados para análise das propriedades psicométricas do instrumento.
Validação do Inventário de Atitudes frente à Dor Crônica - Profissionais Garcia DM, Pimenta CAM, Cruz DALM 
Procedimentos de coleta de dados e aspectos éticos

O estudo foi avaliado pelo Comitê de Ética e Pesquisa de cada uma das Instituições envolvidas. Todos os procedimentos éticos foram observados. Os participantes responderam ao questionário de identificação pessoal e ao Inventário de Atitudes frente a Dor-Profissionais (Pré-teste).

Os questionários foram entregues, no local de trabalho, aos profissionais que concordaram em participar. Em alguns serviços aguardou-se o preenchimento dos mesmos, em outros eles foram recolhidos posteriormente.

\section{Análise dos dados}

Para a confirmação dos fatores foi feita a análise fatorial e a confiabilidade foi estimada por meio do alfa de Cronbach.

\section{RESULTADOS}

\section{Caracterização da amostra}

Foram entrevistados 75 profissionais, o que equivaleu a $59,5 \%$ dos profissionais atuantes nos Centros de Dor. A Tabela 1 apresenta as características da amostra.

Tabela 1 - Caracterização da amostra (n=75) - São Paulo - 2006

\begin{tabular}{|c|c|c|c|c|c|}
\hline Características & $\mathbf{n}$ & \% & Média (dp) & Mediana & Variação \\
\hline \multicolumn{6}{|l|}{ Sexo } \\
\hline Feminino & 38 & 50,7 & & & \\
\hline Masculino & 37 & 49,3 & & & \\
\hline Idade & 75 & & $42,8(10,5)$ & 40 & 24 а 67 \\
\hline Tempo de formado & 75 & & $17,8 \quad(9,9)$ & 16 & 1 а 39 \\
\hline \multicolumn{6}{|l|}{ Profissão } \\
\hline Médico & 44 & 58,7 & & & \\
\hline Enfermeiro & 5 & 6,7 & & & \\
\hline Fisioterapeuta & 11 & 14,7 & & & \\
\hline Psicólogo & 6 & 8,0 & & & \\
\hline Dentista & 8 & 10,7 & & & \\
\hline Assistente Social & 1 & 1,3 & & & \\
\hline \multicolumn{6}{|l|}{ Titulação } \\
\hline Graduação & 22 & 29,3 & & & \\
\hline Especialização & 32 & 42,7 & & & \\
\hline Mestrado & 11 & 14,7 & & & \\
\hline Doutorado & 9 & 12,0 & & & \\
\hline Pós-doutorado & 1 & 1,3 & & & \\
\hline \multicolumn{6}{|c|}{ Auto-avaliação da experiência com dor crônica } \\
\hline Pequena & 12 & 16,0 & & & \\
\hline Média & 45 & 60,0 & & & \\
\hline Grande & 18 & 24,0 & & & \\
\hline \multicolumn{6}{|c|}{$\begin{array}{l}\text { Número de doentes com dor crônica não oncológica } \\
\text { atendidos por mês }\end{array}$} \\
\hline Entre 1 e 5 doentes & 11 & 14,7 & & & \\
\hline Entre 6 e 10 & 12 & 16,0 & & & \\
\hline Entre 11 e 20 & 19 & 25,3 & & & \\
\hline 21 ou mais & 33 & 44,0 & & & \\
\hline
\end{tabular}

Validação do Inventário de Atitudes Frente à Dor - versão profissionais

\section{Análise fatorial}

A análise fatorial teve como finalidade verificar se a estrutura dos fatores mantinha-se semelhante à original após a adaptação do IAD-Breve (doentes) para IADProfissionais

Inicialmente foi feita uma análise com os 7 fatores e os 30 itens originais, através do método de rotação Varimax. Observou-se que vários itens não carregavam no domínio ori- ginal com carga fatorial adequada, que foi definida como acima de $0,3^{(23)}$. Nessa primeira solução, a variância total explicada foi de $57,7 \%$.

Algumas vezes os itens apresentaram carga fatorial mais alta em domínios com os quais não tinham coerência teórica. Visando a ajustar o modelo, analisaram-se as cargas dos itens nos domínios e foram feitos ajustes de modo que os 
itens fossem alocados nos domínios originais desde que apresentassem carga fatorial mínima de 0,3. Se o item carregava com o maior valor no domínio a que originalmente pertencia, foi mantido nesse domínio; se o item carregava no domínio original com carga mínima de 0,3 , foi mantido nesse domínio, mesmo que a carga fatorial tivesse sido maior em outro domínio. A última situação foi quando o item não carregou no domínio original com carga maior que 0,3 e, nesse caso, ele foi excluído, mesmo que ele tivesse carregado com carga alta em outro domínio. Assim, foram excluídos os itens 2, 4, 5, 8, 13, 14, 20 e 21 e o domínio medicação. Com a rotação Varimax e os procedimentos descritos ficaram 22 itens e 6 domínios.
Novas rotações (4) foram realizadas com 6 domínios e 22 itens e a que apresentou melhor solução foi a Equamax, que explicou 63,74\% da variância total. O item 19 precisou ser retirado, pois não atendeu ao critério "apresentar carga fatorial maior que 0,3 no domínio original” e o item 30 foi retirado, pois na análise de confiabilidade observou-se que sua retirada melhorava o alfa de Cronbach do domínio. A versão final ficou com 6 domínios e 20 itens (Anexo 1). A análise fatorial com esses domínios e itens é apresentada na Tabela 2 e a análise de confiabilidade desses domínios, na Tabela 4. A adequação da amostra foi testada pelo KaiserMeyer-Olken (KMO) que foi igual a 0,67 e confirmada pelo Bartlett que foi igual a 0,000 .

Tabela 2 - Análise fatorial, correlações entre itens e 6 domínios, do IAD-profissionais - São Paulo - 2006

\begin{tabular}{lrrrrrr}
\hline \multicolumn{7}{c}{ DOMÍNIOS } \\
Item & $\mathbf{1}$ & $\mathbf{2}$ & $\mathbf{3}$ & $\mathbf{4}$ & $\mathbf{5}$ & $\mathbf{6}$ \\
\hline & $\mathbf{0 , 8 0 6}$ & 0,114 & 0,290 & $-0,038$ & 0,076 & 0,002 \\
10 & $\mathbf{0 , 8 7 7}$ & 0,121 & 0,192 & $-0,017$ & 0,033 & $-0,024$ \\
15 & $\mathbf{0 , 6 9 2}$ & 0,010 & 0,014 & 0,229 & $-0,052$ & $-0,120$ \\
25 & $\mathbf{0 , 7 3 6}$ & $-0,173$ & 0,143 & 0,062 & $-0,258$ & 0,231 \\
11 & $-0,082$ & 0,094 & $-0,140$ & $\mathbf{0 , 7 6 2}$ & 0,157 & 0,149 \\
16 & 0,044 & $-0,072$ & 0,113 & $\mathbf{0 , 4 7 5}$ & 0,474 & 0,189 \\
27 & 0,070 & $-0,111$ & $-0,398$ & $\mathbf{0 , 4 3 8}$ & 0,149 & 0,044 \\
28 & $-0,134$ & 0,219 & $-0,095$ & $\mathbf{0 , 6 8 3}$ & 0,132 & $-0,061$ \\
1 & 0,367 & $-0,116$ & $\mathbf{0 , 3 4 2}$ & 0,024 & 0,359 & 0,091 \\
12 & 0,126 & 0,103 & $\mathbf{0 , 3 4 2}$ & $-0,180$ & 0,056 & 0,051 \\
17 & 0,182 & $-0,043$ & $\mathbf{0 , 6 4 0}$ & $-0,156$ & $-0,111$ & $-0,005$ \\
22 & 0,202 & $-0,071$ & $\mathbf{0 , 7 2 0}$ & 0,060 & 0,338 & 0,022 \\
3 & $-0,088$ & $\mathbf{0 , 8 0 7}$ & 0,023 & 0,089 & 0,069 & 0,131 \\
7 & 0,229 & $\mathbf{0 , 7 7 3}$ & 0,113 & 0,049 & 0,094 & 0,097 \\
9 & 0,010 & $\mathbf{0 , 6 1 7}$ & 0,281 & $-0,261$ & 0,300 & 0,090 \\
18 & $-0,052$ & $\mathbf{0 , 6 8 5}$ & 0,025 & 0,348 & $-0,191$ & 0,078 \\
23 & $-0,065$ & 0,152 & 0,226 & 0,290 & $\mathbf{0 , 7 3 7}$ & 0,084 \\
26 & $-0,067$ & $-0,057$ & $-0,157$ & 0,101 & $\mathbf{0 , 7 7 8}$ & 0,120 \\
24 & 0,054 & 0,115 & $-0,059$ & 0,175 & 0,088 & $\mathbf{0 , 8 2 7}$ \\
29 & $-0,049$ & $-0,066$ & 0,020 & $-0,136$ & 0,026 & $\mathbf{0 , 8 4 7}$ \\
\hline
\end{tabular}

Nota: Domínios 1: emoção 2: solicitude 3: controle 4: dano físico 5: incapacidade 6: cura médica.

Considerando-se a coerência teórica entre domínio e item, a carga fatorial do item no domínio e os valores da análise de confiabilidade (Tabela 4), foram compostos os domínios expressos na Tabela 3. Para a nova composição dos domínios foram excluídos dez itens (2, 4, 5, 8, 13, 14, 19, 20, 21 e 30) e um domínio (medicação). O domínio emoção permaneceu igual à versão original (IAD-Breve). O domínio controle teve o item 20 excluído e o dano físico teve o item 19 excluído; no domínio solicitude foi excluído o item 14; no domínio incapacidade foi excluído o item 30 e no domínio cura médica foram excluídos os itens 4,8 e 21 . Todos os itens do domínio medicação foram excluídos (2, 5, e 13) e esse domínio desapareceu do IAD-profissionais. 
Tabela 3 - Proposição de domínios e itens - São Paulo 2006

\begin{tabular}{lrr}
\hline Domínios & $\begin{array}{c}\text { Itens do IAD - } \\
\text { profissionais }\end{array}$ & $\begin{array}{c}\text { Itens do IAD-breve } \\
\text { doentes (original) }\end{array}$ \\
\hline Emoção & $6,10,15,25$ & $6,10,15,25$ \\
Dano Físico & $11,16,27,28$ & $11,16,19,27,28$ \\
Controle & $1,12,17,22$ & $1,12,17,20,22$ \\
Solicitude & $3,7,9,18$ & $3,7,9,14,18$ \\
Incapacidade & 23,26 & $23,26,30$ \\
Cura Médica & 24,29 & $4,8,21,24,29$ \\
Medicação & - & $2,5,13$ \\
\hline
\end{tabular}

Análise de Consistência

A análise de consistência interna de um instrumento é uma das técnicas para estimar a confiabilidade. O coeficiente alfa de Cronbach é o caso mais geral desse tipo de análise. Com essa técnica verifica-se a congruência que cada item do instrumento tem com o restante dos itens. O coeficiente obtido, que pode variar de 0 a 1, reflete o grau de covariância dos itens entre si, servindo de indicador da consistência interna do domínio/ instrumento.

No IAD-Profissionais, as somas dos escores dos itens componentes de cada domínio representam escores de atitudes/crenças no domínio a que se referem. Para verificar a contribuição de cada item na consistência interna do domínio, considerou-se, principalmente, o efeito da retirada de cada item na confiabilidade. A análise de consistência interna foi realizada para cada domínio e a versão final está apresentada na Tabela 4.

Tabela 4 - Análise de consistência ( $\alpha$ de Cronbach) de cada domínio do IAD - profissionais - São Paulo - 2006

\begin{tabular}{|c|c|c|c|}
\hline Domínio / Item & & m com & $\alpha$ (se item deletado) \\
\hline \multicolumn{4}{|c|}{ Controle $\alpha=0,643$} \\
\hline & 1 & 0,225 & 0,704 \\
\hline & 12 & 0,611 & 0,452 \\
\hline & 17 & 0,430 & 0,571 \\
\hline & 22 & 0,469 & 0,541 \\
\hline \multicolumn{4}{|l|}{ 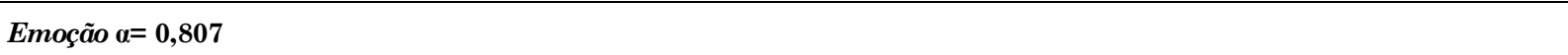 } \\
\hline & 6 & 0,699 & 0,722 \\
\hline & 10 & 0,789 & 0,685 \\
\hline & 15 & 0,490 & 0,834 \\
\hline & 25 & 0,562 & 0,786 \\
\hline \multicolumn{4}{|c|}{ Incapacidade $\alpha=0,688$} \\
\hline & 23 & 0,525 & não se aplica \\
\hline & 26 & 0,525 & não se aplica \\
\hline \multicolumn{4}{|c|}{ Dano Físico $\alpha=0,567$} \\
\hline & 11 & 0,542 & 0,345 \\
\hline & 16 & 0,261 & 0,598 \\
\hline & 27 & 0,281 & 0,552 \\
\hline & 28 & 0,391 & 0,482 \\
\hline \multicolumn{4}{|c|}{ Solicitude $\alpha=0,720$} \\
\hline & 3 & 0,597 & 0,603 \\
\hline & 7 & 0,573 & 0,618 \\
\hline & 9 & 0,375 & 0,729 \\
\hline & 18 & 0,500 & 0,664 \\
\hline \multicolumn{4}{|c|}{ Cura Médica $\alpha=0,595$} \\
\hline & 24 & 0,457 & \\
\hline & 29 & 0,457 & \\
\hline
\end{tabular}


Pela Tabela 4, observa-se que o valor do alfa de Cronbach obtido para o domínio controle foi moderado $(0,643)$. Para o domínio emoção, o valor do alfa de Cronbach foi bom $(0,807)$, mostrando que o domínio é bastante consistente; a exclusão do item 15 poderia aumentar mais o valor de alfa, porém, como o valor com os quatro itens já estava elevado, optouse por não excluí-lo. No domínio incapacidade, o valor do alfa de Cronbach foi considerado moderado $(0,688)$. Para o domínio dano físico, o valor do alfa de Cronbach também foi moderado $(0,567)$. No domínio solicitude, o valor do alfa de Cronbach foi bom $(0,720)$ e no domínio cura médica, o alfa de Cronbach foi moderado $(0,595)^{(24)}$.

\section{DISCUSSÃO}

Para a validação do Inventário de Atitudes Frente à DorProfissionais foram entrevistados 75 profissionais de saúde que atuavam em Centros de Dor da cidade de São Paulo. Desses, 58,7\% eram médicos, 14,7\% fisioterapeutas e 10,7\% dentistas. Observou-se pequena parcela de psicólogos (8\%), enfermeiros $(6,7 \%)$ e assistentes sociais $(1,3 \%)$ nas equipes e isso chamou a atenção, pois, pela etiopatogenia, a avaliação e o tratamento da dor incluem várias modalidades e procedimentos além dos realizados por médicos. A carência desses profissionais pode afetar os resultados terapêuticos.

Encontraram-se enfermeiros apenas em cinco dos nove serviços analisados e isso pode comprometer a qualidade do atendimento. Na dor aguda os enfermeiros são fundamentais para a vigilância e ajuste rápido da terapêutica; na dor crônica, os enfermeiros desenvolvem ações de educação e suporte emocional aos doentes e familiares, implementam estratégias terapêuticas não farmacológicas, padronizam modelos de avaliação e de registro, estabelecem fluxo de comunicação entre os profissionais, atuam para maximizar a adesão ao tratamento, investigam a ocorrência de efeitos colaterais e propõem ações que os evitem ou minimizem.

O IAD-Profissionais foi validado com 6 domínios e 20 itens, conforme pode-se observar nas Tabelas 2,3,4, (o instrumento original possuía 7 domínios e 30 itens). Seguindo os critérios estabelecidos no método, foram excluídos todos itens que não apresentaram carga fatorial acima de 0,3 em seu domínio original e, desse modo, 10 itens e um domínio (medicação) deixaram de existir na versão final do inventário (Anexo 1). Nenhum dos itens do domínio medicação, apresentou carga fatorial adequada no domínio original, como se não houvesse correlação entre eles.

A não confirmação do domínio medicação trouxe indagações. A redação dos itens que se referiam ao tratamento da dor por meio de medicamentos (Provavelmente sempre se terá que utilizar medicamentos para tratar dor crônica; O maio alívio da dor que se tem é com o uso de medicamentos e Medicamentos são um dos melhores tratamentos para dor), aparentemente, estão claros, e parece não ter sido essa a razão da não confirmação desse domínio. É possível que a confusão seja conceitual. O senso comum, o que está mais arraigado entre os profissionais e leigos, é que analgésicos aliviam a dor, o que não é incorreto, especialmente na situação de dor aguda. No entanto, no tratamento da dor crônica não oncológica, embora os medicamentos sejam utilizados e muitas vezes sejam úteis, nem sempre são as intervenções mais eficazes e, nesse caso, não tem sentido utilizá-los por tempo indeterminado. Os profissionais entrevistados possivelmente conheciam esses novos conceitos, mas, provavelmente, ainda conviviam com o conceito da superioridade do remédio para todo tipo de dor e isso levou o mesmo profissional a afirmar essas idéias em algumas questões e a negálas em outra. Disso resultou que não se conseguiu compor o domínio medicação.

Os itens 2,4,5,8,13,14,19,20,21 foram excluídos por não apresentarem carga fatorial adequada no domínio original e o item 30 foi excluído, pois sua retirada melhorava o alfa de Cronbach (de 0,529 para 0,688) no domínio incapacidade. $O$ alfa de Cronbach dos 6 domínios variou de moderado a bom (0,567 a 0,807), o que é considerado suficiente para a mensuração de conceitos subjetivos ${ }^{(24)}$.

A possibilidade de se manterem 6 domínios com confiabilidade adequada indicou que a transposição para profissionais do modelo de crenças/atitudes frente à dor de doentes foi possível, o que sugere coerência desse modelo de crenças frente à dor. Por outro lado, a necessidade de exclusão de 10 itens, do domínio medicação e os valores moderados do alfa de Cronbach nos domínios dano físico e cura médica (0,567 e 0,595 respectivamente) sugerem que o modelo de crenças frente à dor dos doentes para os profissionais talvez possa ser aperfeiçoado. Indaga-se se o problema deveu-se ao ajuste da linguagem ou se os conceitos e crenças dos profissionais sobre dor crônica seriam diferentes dos doentes. O ocorrido com o item 14 do domínio solicitude é um bom exemplo.

O item 14 não teve carga fatorial adequada em solicitude, mas teve carga fatorial adequada em controle. Esse item parece não ter mantido o mesmo significado ao ser adaptado para o IAD-Profissionais. A família precisa aprender a lidar melhor com o doente quando ele está com dor, pressupunha uma resposta tendendo ao totalmente falso, visto querer significar que a família não devia manifestar excesso de solicitude toda vez que o doente sentisse dor e sim estimular o autocuidado, a independência e a movimentação. Porém aprender a lidar melhor com o doente parece que foi entendido que a família deveria ser educada para aprender a lidar com o doente, o que lhe possibilitaria exercer melhor controle sobre a situação.

É interessante notar que alfa de Cronbach moderado foi observado nos domínios dano físico e cura médica e que o domínio excluído foi o de medicação. As crenças representadas nesses domínios diferem das do senso comum de dor
Validação do Inventário de Atitudes frente à Dor Crônica - Profissionais Garcia DM, Pimenta CAM, Cruz DALM 
e de doença. No modelo clássico de dor e de doença, sempre há um dano físico que se espera curar e a cura geralmente se dá por meio de medicamentos e cirurgias. Na dor crônica, o dano físico (existência de uma lesão observável) e a cura no sentido clássico (algo que vai embora e nunca mais volta) nem sempre são possíveis e os remédios nem sempre são bons tratamentos para controlar a dor. Esses conceitos, talvez por diferirem do senso comum e serem relativamente novos, tenham gerado dificuldades de compreensão que influíram na consistência desses domínios.

Embora o estudo tenha trabalhado com as clínicas de dor da cidade de São Paulo, o número de profissionais entrevistados (59,5\%) tenha sido representativo desse universo e o tamanho da amostra ter sido adequada ao estudo, é desejável que o instrumento seja testado em amostras maiores de profissionais especializados em dor e nos não especializados, visto que uma grande gama de profissionais atua com doentes com dor crônica. É interessante que maior número de enfermeiras sejam avaliadas, visto que elas atendem doentes com dor crônica nos ambientes hospitalar, na saúde do trabalhador, nos ambulatórios especializados (ortopedia, reumatologia, etc.). Além disso, estudos que pudessem analisar as crenças sobre medicação em dor crônica, cujo domínio não foi confirmado no presente estudo, são desejáveis.

\section{REFERÊNCIAS}

1. McCaffery M, Pasero C. Pain clinical manual. $2^{\text {nd }}$ ed. St. Louis: Mosby; 1999. Assessment underlying complexities, misconception and pratical tools; p. 35-102.

2. Pimenta CAM. Dor: manual clínico de enfermagem. São Paulo: [s.n.]; 2000.

3. Pimenta CAM, Koizumi MS. Analgesia em câncer: crenças e atualização. Rev Esc Enferm USP. 1993;27(2):309-14.

4. Paice JA. Pain. In: McGuire DB, Yarbro CH. Cancer pain management. $2^{\text {nd }}$ ed. Boston: Jones and Bartlett; 1995. p. 100-25.

5. Brown CA, Richardson C. Nurses' in the multi-professional pain team: a study of attitudes, beliefs and treatment endorsements. Eur J Pain. 2006;10(1):13-22.

6. Ferrell BR, McGuirre DB, Donovan MI. Knowledge and beliefs regarding pain in a sample of nursing faculty. J Prof Nurs. 1993;9(2):79-88.

7. Teixeira MJ, editor. Dor no Brasil: estado atual e perspectivas. São Paulo: Limay; 1995.

8.Glajchen M. Chronic pain: treatment barriers and strategies for clinical practice. J Am Board Fam Pract. 2001;14(3):211-8.

9. Shaw SM. Nursing and supporting patients with chronic pain. Nurs Stand. 2006;20(19):60-5.

10. Lee KF, Ray JB, Dunn GP. Chronic pain management and the surgeon: barries and opportunities. J Am Coll Surg. 2001; 193(6): 689-702.

\section{CONCLUSÃO}

Os profissionais da saúde cuidam dos doentes de acordo com o que conhecem e acreditam, isto é, de acordo com seus conceitos e crenças. Este estudo disponibiliza um instrumento para aferir crenças/atitudes sobre dor crônica entre profissionais de saúde, o que poderá auxiliar na organização e avaliação de ações educativas. O Inventário de Atitudes frente à Dor versão para profissionais foi validado com 20 itens e 6 domínios (emoção, controle, incapacidade, solicitude, cura médica e dano físico); a confiabilidade dos domínios, avaliada por meio do alfa de Cronbach, variou entre 0,56 a 0,80 , valores considerados moderados e bons. Considerando que a maior parte da amostra foi de médicos, há necessidade de estudos que confirmem essas crenças junto a outros profissionais. Os enfermeiros desenvolvem ações educativas junto ao grupo de enfermagem, junto a outros profissionais e clientes. Disponibilizar instrumento que possa aferir conceitos das enfermeiras sobre dor crônica não oncológica propicia adequar as crenças dos enfermeiros e, por conseguinte, prepará-los para educar mais adequadamente os doentes. Estudos que refinem o modelo de mensuração das crenças/atitudes sobre dor crônica entre profissionais de saúde e ampliem a compreensão dos elementos que as determinam e mantêm são desejáveis.

11. Sinatra RS. Acute pain management and acute pain services. In: Cousins MJ, Bridenbaugh PO, eds. Neural blockade: in clinical anesthesia and management of pain. $3^{\text {th }}$ ed. Philadelphia: Raven Press; 1998. p. 793-835

12. Bonica JJ. The management of pain. $2^{\text {nd }}$ ed. Philadelphia: Lea \& Febiger; 1990. History of pain concepts and therapies; p. 2-17.

13. Gallagher RM. Treatment planning in pain medicine. Clin Med North Am. 1999;83(3):823-49.

14. Turkey DC, Meichenbaum D. A cognitive-behavioral approach to pain management. In: Wall P, Melzack R, editor. Textbook of pain. Edinburgh: Churchill Livingstone; 1994. p. 1337-48.

15. Pimenta CAM, Portnoi AG. Dor e cultura. In: Carvalho MM MJ, organizador. Dor: um estudo multidisciplinar. São Paulo: Summus; 1999. p. 159-73.

16. Wright LM, Watson WL, Bell JM. Beliefs: the heart of healing in families and illness. New York: BasicBooks; 1996. Beliefs: many lenses, many explanations; p. 19-43.

17. Beck JS. Terapia cognitiva: teoria e prática. Porto Alegre: Artes Médicas; 1997.

18. Pimenta CAM. Atitudes de doentes com dor crônica frente à dor [tese]. São Paulo: Escola de Enfermagem, Universidade de São Paulo; 1999.

19. Pimenta CAM, Cruz DALM. Crenças em dor crônica: validação do Inventário de Atitudes frente à Dor para a língua portuguesa. Rev Esc Enferm USP. 2006;40(3):365-76. 
20. Tait RC, Chibnall JT. Developmental of a brief version of the Survey of Pain Attitudes. Pain 1997;70(2-3):229-35.

21. Jensen MP, Karoly P, Huger R. The development and preliminary validation of an instrument to assess patients'attitudes toward pain. J Psychosom Res. 1987;31(3):393-400.

22. Castro AB. Tratamento da dor no Brasil: evolução histórica. Curitiba: Ed. Maio; 1999. As clínicas de tratamento de dor no Brasil; p. 74-98.
23. Kline P. An easy guide to factor analysis. London: Routledge; 2000

24. Bowling A. Measuring disease: a review of disease-specific quality of life measurement scales. Philadelphia: Open University Press; 1995. p.292.

Inventário de Atitudes Frente à Dor para profissionais de saúde

\begin{tabular}{|c|c|c|c|c|c|c|}
\hline & \multirow{3}{*}{$\begin{array}{c}\begin{array}{c}\text { Totalmente } \\
\text { Falso }\end{array} \\
0\end{array}$} & & & & \\
\hline & & & \multirow{2}{*}{$\begin{array}{c}\begin{array}{c}\text { Quase } \\
\text { Falso }\end{array} \\
1\end{array}$} & \multirow{2}{*}{$\begin{array}{c}\begin{array}{c}\text { Nem } \\
\text { verdadeiro } \\
\text { Nem Falso }\end{array} \\
2\end{array}$} & \multirow{2}{*}{$\begin{array}{c}\begin{array}{c}\text { Quase } \\
\text { Verdadeiro }\end{array} \\
3\end{array}$} & \multirow{2}{*}{$\begin{array}{c}\text { Totalmente } \\
\text { Verdadeiro } \\
4\end{array}$} \\
\hline 1 & $\begin{array}{l}\text { Muitas vezes o doente consegue influenciar a } \\
\text { intensidade da dor. }\end{array}$ & & & & & \\
\hline 2 & $\begin{array}{l}\text { Sempre que alguém sente dor a família deve } \\
\text { tratá-lo melhor. }\end{array}$ & 0 & 1 & 2 & 3 & 4 \\
\hline 3 & A ansiedade aumenta a dor. & 0 & 1 & 2 & 3 & 4 \\
\hline 4 & $\begin{array}{l}\text { Sempre que alguém sente dor as pessoas devem } \\
\text { tratá-lo com cuidado e preocupação. }\end{array}$ & 0 & 1 & 2 & 3 & 4 \\
\hline 5 & $\begin{array}{l}\text { É responsabilidade dos que amam o doente, } \\
\text { ajudarem-no quando ele sente dor. }\end{array}$ & 0 & 1 & 2 & 3 & 4 \\
\hline 6 & O estresse aumenta a dor. & 0 & 1 & 2 & 3 & 4 \\
\hline 7 & $\begin{array}{l}\text { Exercício e movimento são bons para o doente } \\
\text { com dor. }\end{array}$ & 0 & 1 & 2 & 3 & 4 \\
\hline 8 & $\begin{array}{l}\text { Consegue-se diminuir a dor através da } \\
\text { concentração ou relaxamento. }\end{array}$ & 0 & 1 & 2 & 3 & 4 \\
\hline 9 & A depressão aumenta a dor. & 0 & 1 & 2 & 3 & 4 \\
\hline 10 & O exercício pode piorar ainda mais a dor. & 0 & 1 & 2 & 3 & 4 \\
\hline 11 & $\begin{array}{l}\text { Pode-se controlar a dor mudando-se os } \\
\text { pensamentos. }\end{array}$ & 0 & 1 & 2 & 3 & 4 \\
\hline 12 & $\begin{array}{l}\text { Muitas vezes, quando se está com dor, precisa- } \\
\text { se receber mais carinho que se está recebendo. }\end{array}$ & 0 & 1 & 2 & 3 & 4 \\
\hline 13 & $\begin{array}{l}\text { Pode-se, com certeza, aprender a lidar com a } \\
\text { dor. }\end{array}$ & 0 & 1 & 2 & 3 & 4 \\
\hline 14 & $\begin{array}{l}\text { A dor não impede de se levar uma vida } \\
\text { fisicamente ativa. }\end{array}$ & 0 & 1 & 2 & 3 & 4 \\
\hline 15 & A dor física nunca será curada. & 0 & 1 & 2 & 3 & 4 \\
\hline 16 & $\begin{array}{l}\text { Há uma forte ligação entre as emoções e a } \\
\text { intensidade da dor. }\end{array}$ & 0 & 1 & 2 & 3 & 4 \\
\hline 17 & $\begin{array}{l}\text { A pessoa com dor pode fazer quase tudo que } \\
\text { fazia antes de ter dor }\end{array}$ & 0 & 1 & 2 & 3 & 4 \\
\hline 18 & $\begin{array}{l}\text { Se a pessoa com dor não fizer exercícios } \\
\text { regularmente a dor continuará a piorar. }\end{array}$ & 0 & 1 & 2 & 3 & 4 \\
\hline 19 & $\begin{array}{l}\text { A prática de exercícios pode diminuir a } \\
\text { intensidade da dor. }\end{array}$ & 0 & 1 & 2 & 3 & 4 \\
\hline 20 & Não há procedimento médico que ajude na dor. & 0 & 1 & 2 & 3 & 4 \\
\hline
\end{tabular}

\section{Agradecimentos}

Aos profissionais do Grupo de Pesquisa CNPq Dor, Controle de Sintomas e Cuidados Paliativos, que auxiliaram no refinamento do instrumento e aos profissionais dos Centros de Dor que responderam ao questionário. 